\title{
Local drug delivery to a human pancreatic tumor via a newly designed multiple injectable needle
}

\author{
KOJI OHARA, MASAYUKI KOHNO, TOMOHISA HORIBE and KOJI KAWAKAMI \\ Department of Pharmacoepidemiology, Graduate School of Medicine and Public Health, \\ Kyoto University, Kyoto 606-8501, Japan
}

Received September 24, 2012; Accepted October 26, 2012

DOI: $10.3892 / \mathrm{mco} .2012 .47$

\begin{abstract}
Endoscopic ultrasound-guided fine needle injection (EUS-FNI) has been proposed as a novel technique for local delivery of antitumor agents to refractory tumors, including pancreatic cancer. However, the present outcome of this strategy remains insufficient, and further improvements as well as novel agents and injection devices are required. The aim of the study was to determine the feasibility of a newly designed 'multiple injectable needle' (MIN) for EUS-FNI and the resulting improvements in the drug distribution to the tumor in comparison with straight-type needles. Human pancreatic cancer BxPC-3 cells or orthotopic tumor were transplanted subcutaneously into athymic rats. Ethanol was injected into subcutaneous tumors using a MIN or straighttype needle. Sequential sections of subcutaneous tumors were stained with hematoxylin and eosin, and tumors and injury areas were quantified using ImageJ software. In the orthotopic tumors, injection of the outer needle of the MIN, advancement of the inner needle and ethanol infusion were monitored by EUS. Injury volume of subcutaneous tumors using a MIN was greater than that with the straight-type needles, except for large tumors. Injecting process using MIN was monitored with endoscopic ultrasound (EUS). This study showed improvement in the drug distribution in a tumor, following injection with MIN compared to straight-type needles, suggesting the novel device to be feasible for use in EUS-FNI.
\end{abstract}

\section{Introduction}

Endoscopic ultrasound (EUS) has been used in the diagnosis and staging of pancreatic cancer. Therapeutic studies of pancreatic cancer using EUS include approaches such as photodynamic therapy $(1,2)$ and brachytherapy (3). Radiofrequency

Correspondence to: Professor Koji Kawakami, Department of Pharmacoepidemiology, Graduate School of Medicine and Public Health, Kyoto University, Yoshidakonoecho, Sakyo-ku, Kyoto 606-8501, Japan

E-mail: kawakami.koji.4e@kyoto-u.ac.jp

Key words: endoscopic ultrasound-guided fine needle injection, animal study, pancreatic tumor ablation using needles has also been investigated for its feasibility and safety in normal porcine pancreas (4). EUS-guided fine needle injection (EUS-FNI) was previously introduced as a novel technique for the local delivery of antitumor agents including ethanol, OncoGel (5) and ONYX-105 (6,7). However, studies have yet to show satisfactory antitumor activity against pancreatic cancer.

Development and improvement of new types of needles for EUS-aided drug injection are likely to create new treatment methods for refractory tumors, including pancreatic cancer. The aim of this study was to demonstrate the feasibility of a newly designed 'multiple injectable needle' (MIN) to improve drug distribution within the tumor.

\section{Materials and methods}

Design of the MIN. The MIN, newly designed for this study, is a 25-gauge inner needle embedded in a 19-gauge outer needle (Fig. 1A). The inner needle is advanced from a hole located $7.5 \mathrm{~mm}$ from the outer needle tip. MIN is capable of injecting the drug into the tumor in multiple directions through combined manipulation of the inner needle and rotation of the outer needle at a puncture site (Fig. 1B).

Comparison of ethanol injection into a subcutaneous tumor using three different types of needle in a human pancreatic cancer xenograft model. Animal experiments in the present study were approved by the Animal Research Committee of the Graduate School of Medicine, Kyoto University (MedKyo 12074). Male athymic nude rats $(n=32 ; 4$ weeks old) purchased from CLEA Japan, Inc. (Tokyo, Japan) were used in this study.

Human BxPC-3 pancreatic cancer cells ( $1 \times 10^{7}$ cells) (DS Pharma Biomedical Co., Ltd., Osaka, Japan) resuspended in $150 \mu \mathrm{l}$ of PBS were transplanted subcutaneously into the flank region of nude rats. After 6 weeks, tumors were measured with a caliper and the tumor volume $\left(\mathrm{mm}^{3}\right)$ was calculated using the formula: length $\mathrm{x}$ width ${ }^{2} \mathrm{x} 0.5$. Animals were randomized into 3 groups $(\mathrm{n}=10)$.

After piercing the tumor with the MIN, the inner needle was advanced and ethanol (99.5\%) (Nacalai Tesque Inc., Kyoto, Japan) was slowly infused over a period of $30 \mathrm{sec}$ with a KDS-100 syringe pump (KD Scientific Inc., New Hope, PA, USA). After retracting the inner needle, the needle shaft was rotated $90^{\circ}$, the inner needle was advanced again and an 


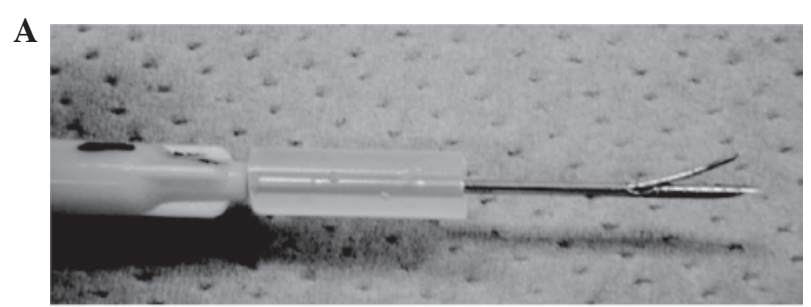

B

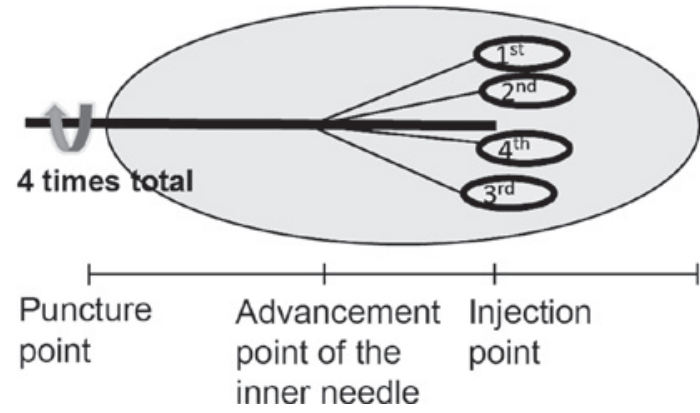

Figure 1. (A) Multiple injectable needle (MIN). (B) Schema of multipoint injection.

additional $50 \mu \mathrm{l}$ of ethanol was injected. Injection was performed 4 times with a total of $200 \mu \mathrm{l}$ of ethanol being injected into the tumor, as shown in Fig. 1B. The same volume (200 $\mu$ l) of ethanol was injected using a single-end-hole 19-gauge needle (conventional needle; THERUMO Corp., Tokyo, Japan) and a 4-side-hole needle (4SHN; EUSN-20-CPN; Cook Medical Inc., Bloomington, IN, USA), under the same conditions.

The rats were sacrificed 7 days after ethanol injection. Tumors were then excised and fixed in formalin, sectioned perpendicular to the needle axis at 0.5 -mm intervals and stained with hematoxylin and eosin (H\&E). Images of the sections were captured using a VS120 scanning system (Olympus Corp., Tokyo, Japan) and boundaries of the injury area were determined within the image. The injury area was defined as the presence of necrosis, fibrosis and regeneration. Tumors and the injury areas were quantified with the ImageJ software.

The injury volume $\left(\mathrm{V}, \mathrm{mm}^{3}\right)$ was calculated using the sum of the injury area in each section $[\mathrm{S}(\mathrm{x})]$, the number of sections $(\mathrm{N})$ and the distance between each section $(0.5 \mathrm{~mm})$ :

$$
V=\frac{1}{2} \sum_{i=1}^{N} S\left(\frac{i}{2}\right)
$$

Ultrasound observations of ethanol injection with MIN in an orthotopic pancreatic tumor model. Two orthotopic model of human pancreatic tumor xenografts in rats were prepared using BxPC-3 cells, as described previously (8). Ten weeks after transplantation, ultrasound observations of ethanol injection into pancreatic tumors using the MIN were performed under anesthesia with an open surgical approach, in which the needle was inserted through the gastric wall under ultrasound guidance (GF-UCT2000-OL5, EU-C2000; Olympus Medical Systems Corp., Tokyo, Japan).

\section{Results}

Comparison of the injury area in subcutaneous tumors following ethanol injection with three types of needle. To evaluate ethanol distribution following injection with three types of needle, sequential sections of subcutaneous tumors were prepared with H\&E staining. As shown in Fig. 2A, MIN resulted in a cross-shaped injury area. However, 4SHN produced an ellipsoidal distribution (Fig. 2B) and the conventional needle produced a circular distribution (Fig. 2C). The range of injury volume was wide in each group, although the mean injury volume with MIN was greater compared to $4 \mathrm{SHN}$ (Fig. 3A). Tumor volume analysis was therefore performed on the day of ethanol injection using the Ward method (JMP8 software; SAS Institute Inc., Cary, NC, USA), a mathematical classification based on the distance among the values (Fig. 3B and $C)$. In the cluster group for large tumors $(n=4)$ (Fig. $3 C$ ), the difference among the three needles was small. However, in the cluster group for small tumors $(\mathrm{n}=6)$ (Fig. 3B), the difference between the MIN and straight-type needles increased.

Ultrasound observation of ethanol injection into an orthotopic tumor with MIN. EUS was used to observe ethanol injection into pancreatic orthotopic tumors using the MIN. Insertion of the outer needle into the tumor, advancement of
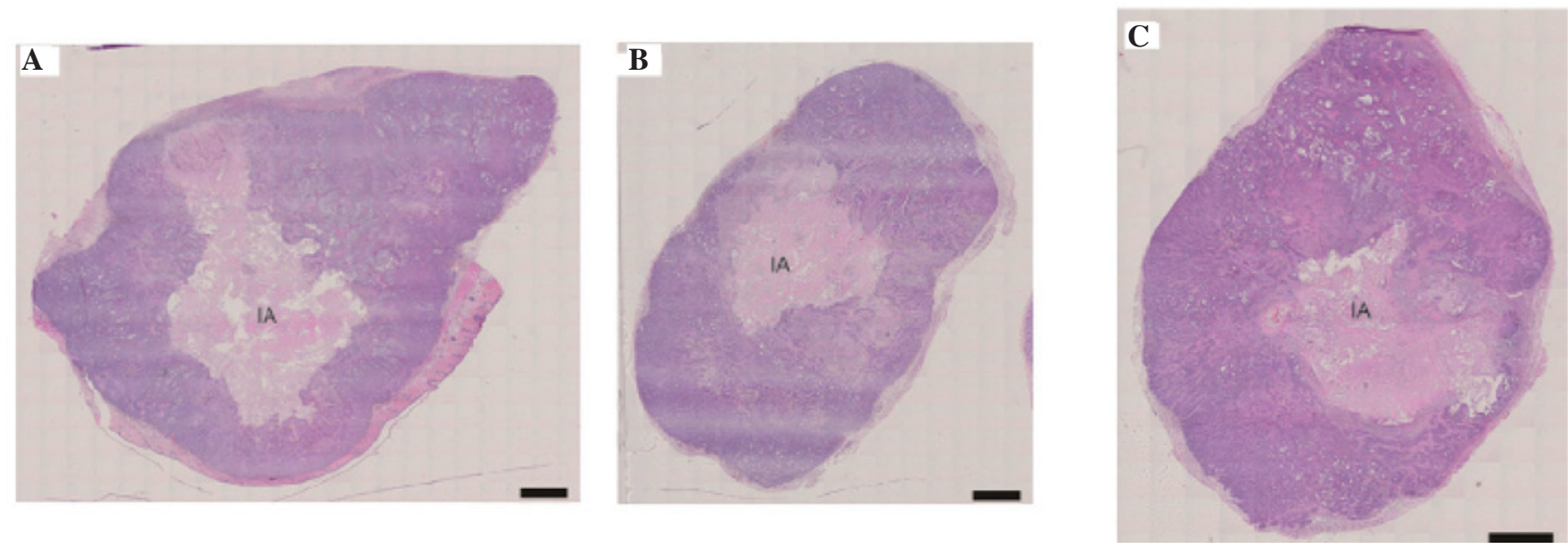

Figure 2. Hematoxylin and eosin-stained images of ethanol distribution with (A) the MIN, (B) a 4-side-hole needle and (C) a conventional needle. IA, injury area;bars, $2 \mathrm{~mm}$. 
A

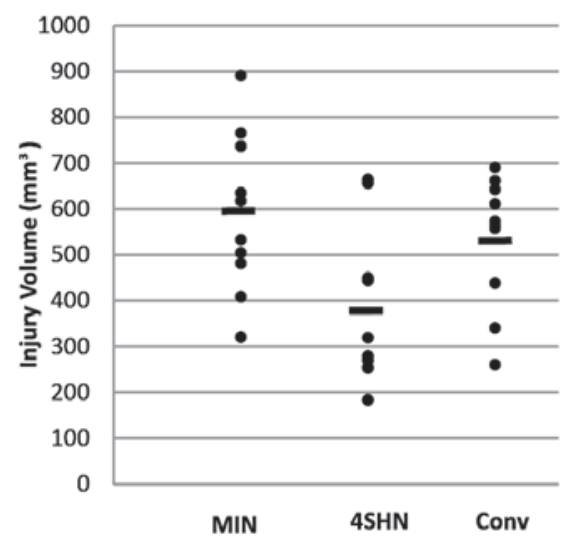

B

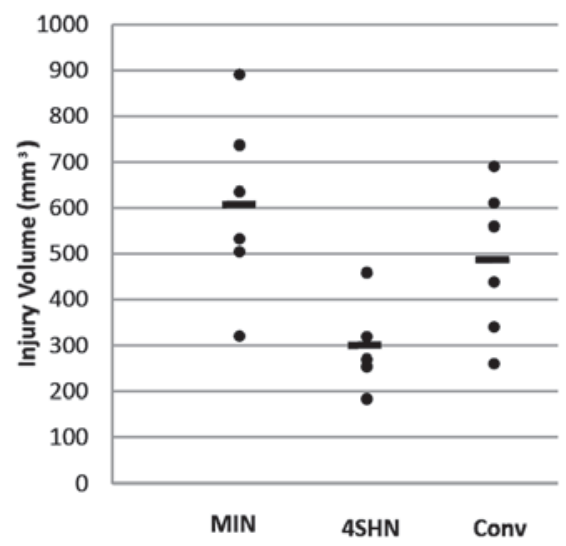

C

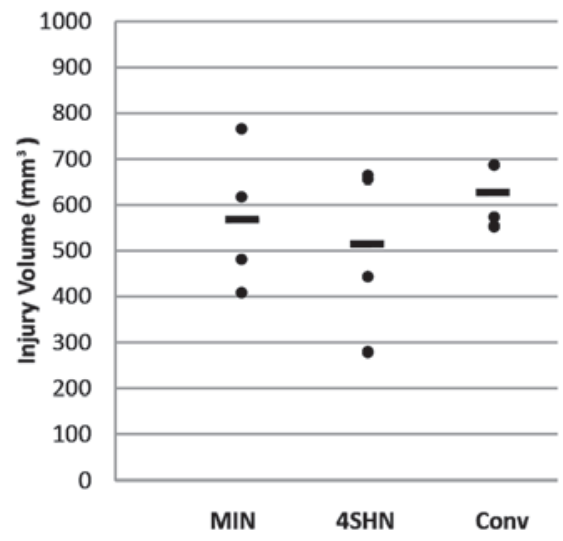

Figure 3. Comparison of the volume of injury with the three types of needle. (A) Overall plot (tumors, $\mathrm{n}=10$ ); plot of the cluster group for (B) small tumors $(n=6)$ and $(C)$ large tumors $(n=4) .4 S H N$, 4-side-hole needle; Conv, conventional needle; bars, the mean.

A

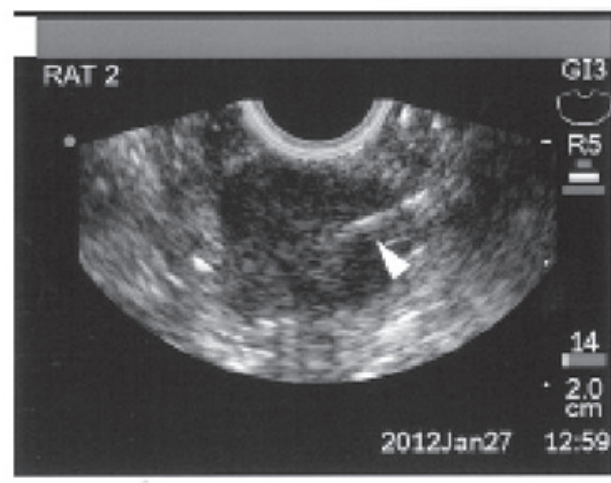

B

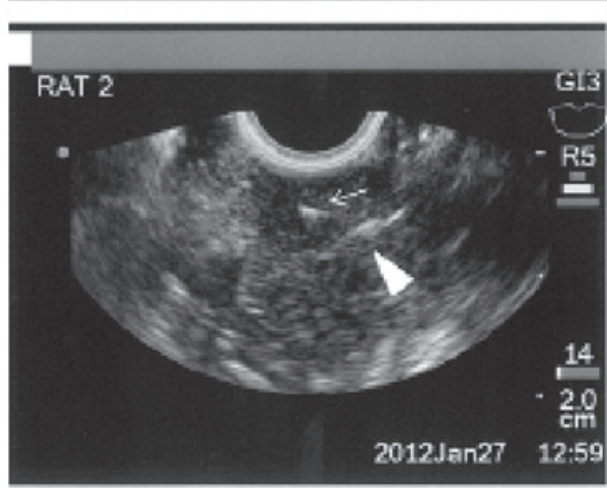

C

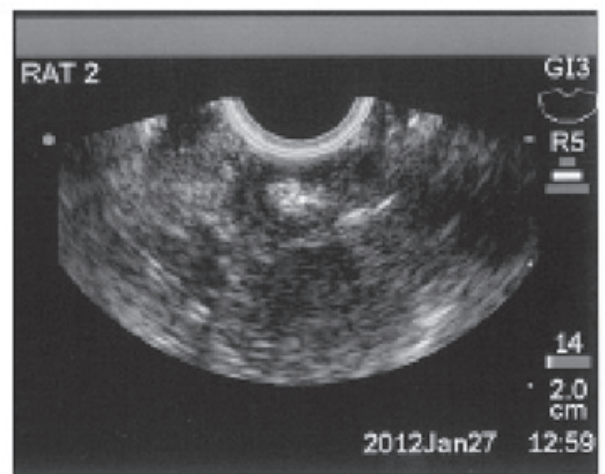

Figure 4. Ultrasound monitoring of ethanol injection into an orthotopic tumor using the multiple injectable needle (MIN). (A) Insertion of the outer needle; (B) advancement of the inner needle and (C) ethanol injection. Arrowheads, outer needle; arrows, inner needle. the inner needle from the outer needle and ethanol infusion were observed on ultrasound images and were visualized as a highly echoic image (Fig. 4).

\section{Discussion}

This study has investigated the effect of structurally different needles on the injury volume in a tumor following the injection of ethanol. As shown in Fig. 2, the injury area showed different forms, depending on the structure of the needle employed. Using MIN, the injury area showed a cross-like distribution. However, with a conventional needle, the distribution was more circular (Fig. 2A). These results indicate that ethanol injected via the MIN was distributed in four directions within the tumor.

The injury volume in the tumor among the three needle types was compared, and the findings showed relatively widespread values (Fig. 3A, n=10). Since large tumors tend to induce inner necrosis, we assumed this would affect the evaluation of the injury volume after injection, and therefore, performed tumor volume analysis on the day of ethanol injection using the Ward method. In the cluster group for small tumors $(n=6)$, the difference between MIN and the straight-type needles increased. Notably, this result was similar to the results of a previous study comparing the distribution of drug injection into bovine liver with a conventional, three-side-hole and multipronged needles (9).

With the MIN, the injury volume was larger compared to $4 \mathrm{SHN}$. This was possibly the result of two factors. First, multipoint injection with advancement of the inner needle enables separation of the needles from the injection point, as well as increasing the area of contact between the tumor and the injected ethanol. Although the distribution of injected ethanol is obstructed by intratumoral barriers such as the septa, advancement of the inner needle may have enhanced the perfusion of ethanol. Second, it is possible that multipoint injection with the MIN may have increased pressure at the injection point, thus affecting the distribution of ethanol compared with four-directional injection within one area with the 4SHN.

In conclusion, the findings suggest that the newly-designed MIN increases the distribution of injected drugs compared 
to straight-type needles, and is therefore of potential use in EUS-FNI as a novel accessory of pancreatic cancer therapy.

\section{References}

1. Bown SG, Rogowska AZ, Whitelaw DE, Lees WR, Lovant LB, Ripley P, et al: Photodynamic therapy for cancer of the pancreas. Gut 50: 549-557, 2002.

2. Chan HH, Nishioka NS, Mino M, Lauwers Gy, Puricelli WP, Collier KN, et al: EUS-guided photodynamic therapy of the pancreas: a pilot study. Gastrointest Endosc 59: 95-99, 2004.

3. Sun S, Xu H, Xin J, Liu J, Guo Q and Li S: Endoscopic ultrasound-guided interstitial brachytherapy of unresectable pancreatic cancer: results of a pilot trial. Endoscopy 38: 399-409, 2006.

4. Gordberg SN, Mallery S, Gazelle GS and Brugge WR: EUS-guided radiofrequency ablation in the pancreas: results in a porcine model. Gastrointest Endsc 50: 392-401, 1999.
5. Matthes K, Mino-Kenudson M, Sahani DV, Holalkere N, Fowers KD, Rathi R, et al: EUS-guided injection of paclitaxel (OncoGel) provides therapeutic drug concentrations in the porcine pancreas (with video). Gastrointest Endosc 65:448-453, 2007.

6. Habib N, Salama H, Abd ELAMA, Isac AI, Abd AARA, Sarraf C, et al: Clinical trial of E1B-deleted adenovirus (d11520) gene therapy for hepatocellular carcinoma. Cancer Gene Ther 9: 254-259, 2002

7. Hecht JR, Bedford R, Abbruzzese JL, Lahoti S, Reid TR, Soetikno RM, et al: A phase I/II trial of intratumoral endoscopic ultrasound injection of ONYX-015 with intravenous gemcitabine in unresectable pancreatic carcinoma. Clin Cancer Res 9: 555-561, 2003

8. Haramoto M, Kohno M, Nakajima O, Horibe T, Kiyohara M, Fukazawa $\mathrm{H}$, et al: Pancreatic cancer therapy with a novel pump for controlled drug release. Oncol Rep 23: 365-370, 2010.

9. Sudheendra D, Léger R, Groppo ER, Sun D, Durrani AK, Neeman Z, et al: Comparison of three different needles for percutaneous injections. Cardiovasc Intervent Radiol 30: 151-152, 2007. 